03,10

\title{
Каналирование медленных ионов в монокристаллическом кремнии
}

\author{
(C) А.Б. Свечников \\ Научно-исследовательский центр „Курчатовский институт“, \\ Москва, Россия \\ E-mail: asvech@mail.ru
}

Поступила в Редакцию 22 июня 2020 г.

В окончательной редакции 22 июня 2020 г.

Ппринята к публикации 6 августа 2020 г.

\begin{abstract}
Профили распределения ионов $\mathrm{Si}^{+}$по глубине монокристаллического кремния рассчитаны методом молекулярной динамики. Неупругие потери энергии при торможении определены в рамках теории функционала электронной плотности. Выполнен анализ факторов, оказывающих влияние на процесс каналирования ионов. В частности, подтверждено существование „эффекта массы“ для критического угла каналирования.
\end{abstract}

Ключевые слова: каналирование ионов, молекулярная динамика, торможение.

DOI: 10.21883/FTT.2020.12.50208.135

\section{1. Введение}

Процессы торможения быстрых ионов в твердых телах представляют большой практический интерес в области ионной имплантации полупроводниковых материалов. Серьезные проблемы создают быстрые атомы отдачи, возникающие в стенках ядерных реакторов под воздействием нейтронов, приводя к радиационным повреждениям. Ионы после потери кинетической энергии и остановки формируют профили распределения по глубине, которые наблюдают экспериментально или получают в модельных расчетах. Тормозные потери ионов принято разделять на ядерные и электронные, вследствие упругих соударений иона с ядрами и неупругих потерь на электронах атомов твердого тела соответственно. Физическая природа данных типов торможения настолько различна, что теоретикам приходится решать две отдельные задачи. Экспериментальные данные относятся к полным потерям энергии иона, и публикуемые вклады отдельных типов торможения носят оценочный характер [1].

Профили распределения ионов по глубине в аморфных телах и кристаллических структурах произвольной ориентации имеют одинаковый вид, что является следствием одинаковой природы процессов торможения в среде, характеризуемой атомным составом и плотностью. В начале 1960-х годов экспериментально и теоретически было показано [2,3], что симметрия кристаллической решетки может быть причиной заметного увеличения длин пробега ионов, т. е. анизотропией торможения ионов, получившей название каналирование. Физически каналирование связывают с перемещением иона в области пониженной электронной плотности атомов решетки и низкими упругими потерями. В каналах снижается вероятность рассеяния иона на атомах решетки под большими углами. Череда „мягких“ столкновений удерживает ион в канале, приводя к удлинению трека в разы по сравнению с торможением в том же кристалле, но в случайном направлении. Различают осевое и планарное каналирование, в зависимости от типа симметрии кристаллической решетки и направления движения иона.

В качестве иллюстрации на рис. 1 показано распределение электронной плотности в виде изоповерхности для грани $\langle 100\rangle \mathrm{Si}$, рассчитанной нами в приближении функционала электронной плотности (модифицированный код abinit [4]). Можно наблюдать отверстия в изоповерхности, совпадающие с „пустотами“ в решетке, через которые может осуществляться осевое каналирование ионов.

Настоящая работа посвящена моделированию процесса каналирования методом молекулярной динамики. Рассматриваются профили распределения ионов кремния в монокристаллическом кремнии с ориентацией $\langle 100\rangle$ при низких дозах облучения. Основное внимание

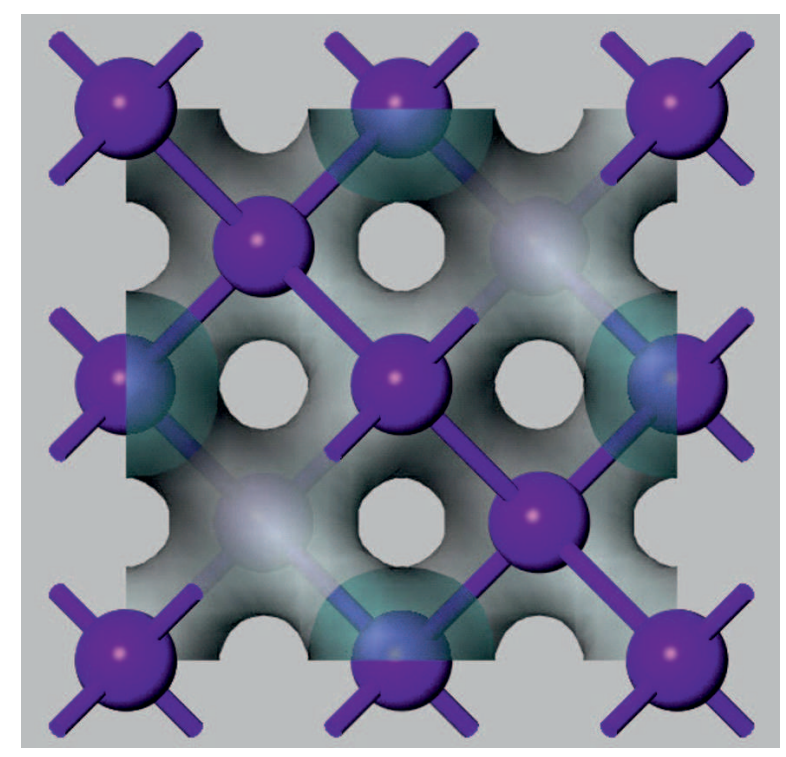

Рис. 1. Изоповерхность плотности электронов (величина 0.07$)$ для $\langle 100\rangle \mathrm{Si}$. 
уделяется физическим факторам, являющимся помехой для каналирования ионов.

Отметим, что большинство авторов, ссылки на работы которых можно найти в цитируемой литературе, рассматривает каналирование в кремнии с целью оптимизации процесса легирования, где первую роль играют ионы бора и фосфора. По нашему мнению, медленные ионы могут быть использованы для инициализации структурных изменений на определенных глубинах от поверхности монокристаллической грани. Представленные ниже профили распределения ионов дают возможность проанализировать эволюцию структурных дефектов в монокристаллическом кремнии, однако эта задача выходит за рамки данной работы. Также было учтено практически полное отсутствие литературных данных по каналированию ионов кремния.

В основу моделирования легла методика неэмпирического расчета электронных тормозных потерь, объединенная с решением классической задачи упругого рассеяния на экранированном кулоновском потенциале. С помощью многочастичного потенциала воспроизводилась динамика столкновений и теплового движения атомов решетки кремния. Траектория движения иона в кристаллической структуре рассчитывалась путем интегрирования классических уравнений движения по стандартной схеме предиктор-корректор. Шаг интегрирования варьировался в зависимости от текущей кинетической энергии иона, например, для $10 \mathrm{keV}$ имел значения порядка $10^{-17}-10^{-16} \mathrm{~s}$. Для построения отдельного профиля распределения ионов по глубине выполнялся расчет 10000 треков, местом остановки иона являлось положение, где кинетическая энергия иона была меньше $1 \mathrm{eV}$. График профиля представляет сглаженную гистограмму с числом интервалов порядка 200.

Анализ профиля распределения каналирования ионов показал наличие трех групп ионов с характерными особенностями динамики торможения. На основе сравнения профилей распределения проанализирована восприимчивость эффекта осевого каналирования к температуре кристалла, углу падения иона на поверхность и начальной энергии иона. В заключении рассмотрен вопрос о влиянии массы иона на характер каналирования, т.н. изотопный эффект, вызывающий споры среди исследователей.

\section{2. Методика расчета}

\section{1. Геометрия}

Кристаллическая структура решетки кремния без примесей и дефектов с ориентацией $\langle 100\rangle$ моделировалась ячейкой порядка 100 атомов. Влияние температуры на решетку учитывалось с помощью [5]:

$$
\left\langle\Delta r^{2}\right\rangle=\frac{9 \hbar T}{M k T_{\mathrm{D}}^{2}},
$$

где $\left\langle\Delta r^{2}\right\rangle$ - среднее смещение атомов из узлов идеальной решетки, $T-$ температура решетки, $T_{\mathrm{D}}=645 \mathrm{~K}$ соответствует температуре Дебая для кремния, $M$ масса атома кремния, $\hbar$ и $k-$ постоянные Планка и Больцмана. Поправки к координатам атомов рассчитывались с помощью функции $\left\langle\Delta r^{2}\right\rangle$ и случайных чисел.

Выбор граничных условий обеспечивал перемещение иона в решетке без дефектов, а для сохранения размера ячейки и экономии памяти часть атомов решетки позади иона удалялась.

Начальная высота иона над кристаллом составляла несколько ангстрем, две остальные координаты в плоскости грани, выбирались случайным образом. Величина начальной скорости иона соответствовала выбранной энергии, направление составляло фиксированный угол $(\alpha)$ с нормалью к поверхности. Для оптимального каналирования требуется выбор $\alpha=0^{\circ}$.

\section{2. Взаимодействие}

В монокристаллическом кремнии атомы имеют характерное тетраэдрическое окружение. Исследования показали, что с помощью лишь двухатомных (парных) потенциалов взаимодействия воспроизвести данную структуру не удается, необходимо добавление трехатомных вкладов. В настоящей работе использовался зависящий от окружающей среды межатомный потенциал (EDIP) Базанта-Кашираса [6]:

$$
V_{\mathrm{Si}-\mathrm{Si}}=V_{2}+V_{3}
$$

где $V_{2}$ - парные вклады, зависящие от межатомных расстояний и функции координационного числа, $V_{3}$ трехатомные вклады также зависят от углов между атомными связями. Опыт использования потенциала EDIP показал его высокую точность в описании кристаллических и аморфных структур, однако он требователен к производительности вычислений.

Ион-атомные столкновения моделировались универсальным потенциалом отталкивания [7]:

$$
V_{i-\mathrm{Si}}=\frac{Z_{i} Z_{\mathrm{Si}} e^{2}}{4 \pi \varepsilon_{0} r} \varphi\left(\frac{r}{a}\right),
$$

где $\varphi(x)$ - функция экранирования, $Z_{i}, Z_{\mathrm{Si}}$ - атомные номера иона и атома среды $(\mathrm{Si}), e-$ заряд электрона, $r$ - межъядерное расстояние, $a$ - длина экранирования

$$
a=\frac{0.4685}{Z_{i}^{0.23}+Z_{\mathrm{Si}}^{0.23}} .
$$

Потенциал $V_{i-\mathrm{Si}}$ используется в приложении TRIM [7] для вычисления углов рассеяния ионов низких энергий, когда резерфордовское приближение дает плохие результаты.

\section{3. Неупругие потери}

В рамках современной теории не существует единого универсального метода определения неупругих потерь 
энергии ионом $\left(E_{i}\right)$ в твердом теле для всего спектра энергий. В литературе различают следующие ионы: 1) быстрые $V_{i}>v_{0} Z_{i}^{2 / 3}$ и $V_{i}>v_{0} Z_{\mathrm{Si}}^{2 / 3}$;2) промежуточные $\left.V_{i} \approx v_{0} \approx v_{\mathrm{F}} ; 3\right)$ медленные $V_{i}<v_{0}$, где $V_{i}-$ скорость иона, $v_{0}$ - боровская скорость, $v_{\mathrm{F}}$ - скорость Ферми атома среды. Для иона $\mathrm{Si}^{+}$в кристаллическом $\mathrm{Si}$ имеем $Z_{i}=Z_{\mathrm{Si}}=14, \quad v_{\mathrm{F}}=2.131 \cdot 10^{6} \mathrm{~m} / \mathrm{s}, \quad v_{0}=2.188 \cdot 10^{6} \mathrm{~m} / \mathrm{s}$. Определим типичные значения энергий для быстрых, промежуточных и медленных ионов $\mathrm{Si}^{+}$и рассмотрим методы расчета электронного торможения.

Ионы Si c промежуточными энергиями около $0.66 \mathrm{MeV}$ использовались нами в небольшом количестве расчетов, рассмотрим их неупругое торможение подробнее.

Быстрые ионы $\mathrm{Si}$ с энергиями $23 \mathrm{MeV}$ и выше представляют собой голые ядра с зарядом $Z_{i} e$, неупругие потери описываются теорией Бете с поправкой Блоха. В настоящей работе быстрые ионы не рассматривались.

Ионы $\mathrm{Si}$ с промежуточными энергиями около $0.66 \mathrm{MeV}$ торможение подробнее. Предполагается, что ион заряжен частично, и часть электронов ближайшего к иону атома среды находится в возбужденном состоянии. В работе использовалось полуэмпирическое приближение Циглера-Бирсака-Литтмарка (ZBL, [7]). Пусть ион несет на себе $\mathrm{N}$ электронов, определим степень ионизации иона $q=Q / Z_{i}$, где $Q=\left(Z_{i}-N\right) \cdot e-$ ионное зарядовое число. Используя известные данные по потерям протонов с той же энергией в той же среде $\left(S_{p}\right)$, определяют потери иона $(S)$ с помощью „правила масштабирования для тяжелых ионов“

$$
S=\gamma Z_{i}^{2} S_{p}
$$

и вычисляют $\gamma$ - эффективный заряд иона, зависящий от степени ионизации и размера иона $(\Lambda)$ :

$$
\begin{gathered}
\gamma=q+\frac{(1-q) v_{0}^{2}}{2 v_{\mathrm{F}}^{2}} \ln \left[1+\left(\frac{4 \Lambda v_{\mathrm{F}}}{1.919}\right)^{2}\right], \\
\Lambda=\frac{2 a a_{0}\left(\frac{N}{Z_{i}}\right)^{2 / 3}}{Z_{i}^{1 / 3}\left(1-\lambda \frac{N}{Z_{i}}\right) / 4},
\end{gathered}
$$

где $a=0.24, a_{0}=0.529 \cdot 10^{-8} \mathrm{~cm}, \lambda=4 / 7$.

$\mathrm{B}$ основе приближения лежит теория линейного отклика, где сначала находят заряды, наведенные на атоме среды, а затем вычисляют тормозную кулоновскую силу, действующую обратно на ион. Несколько грубо в теории выглядит учет динамического отклика от газа свободных электронов, однако, привлечение экспериментальных данных значительно улучшает результаты. Приближение ZBL реализовано в приложении TRIM [7].

Медленные ионы $\mathrm{Si}$ с энергиями заметно ниже $0.66 \mathrm{MeV}$ (нами использовался верхний порог в $0.4 \mathrm{MeV}$ ) представляют собой практически нейтральные атомы $\mathrm{Si}$, причем валентные электроны ближайшего к иону атома $\mathrm{Si}$ среды могут быть возбуждены. Квазиклассические теории торможения Фирсова [8] и группы Линдхарда [9] несмотря на упрощенное описание взаимодействия иона с атомом среды успешно воспроизводили линейную зависимость $S$ от $V_{i}$. Однако впоследствии выяснилось, что зависимость не является линейной [10].

Рассмотрим схему строгого расчета неупругого торможения в рамках неэмпирических квантовых расчетов электронной структуры медленных ионов и окружающих атомов среды. Начальный этап включает расчет пространственного распределения электронной плотности $n(r)$ в монокристаллическом Si. Мы воспользовались модифицированным кодом abinit [4] в приближении локальной плотности теории функционала электронной плотности с обобщенной градиентной аппроксимацией Perdew-Burke-Ernzerhof (PBE GGA) [11] для обменнокорреляционной энергии. Не вдаваясь в сложный анализ, отметим, что точный вид $n(r)$ очень важен при расчете неупругих потерь, не следует экономить с однородным [12] и сферически симметричным [13] распределениями электронной плотности.

Далее будем следовать в русле модели торможения, изложенной авторами [13]. Ключевым моментом будет использование функции одноэлектронного радиуса $\left(r_{s}\right)$, которая связана с электронной плотностью и скоростью Ферми следующим образом:

$$
\frac{4 \pi}{3} r_{s}(r)^{3}=\frac{1}{n(r)}, \quad r_{s}(r)=\frac{v_{\mathrm{F}}(r)}{1.919} .
$$

Теперь неупругие потери [8] для неоднородного распределения электронов имеют вид

$$
S=\frac{3 V_{i}}{v_{\mathrm{F}} r_{S}(r)^{3}} \sum_{L}(L+1) \sin ^{2}\left[\delta_{L}\left(v_{\mathrm{F}}\right)-\delta_{L+1}\left(v_{\mathrm{F}}\right),\right.
$$

где $L-$ индекс парциальной волны, $\delta_{L}\left(v_{\mathrm{F}}\right) \equiv \delta_{L}\left(v_{\mathrm{F}}(r)\right)-$ фазовый сдвиг для рассеянного электрона на поверхности Ферми, который находится из асимптоты волновой функции

$$
u_{L} \rightarrow A_{L} \sin \left(k r-\frac{\pi L}{2}+\delta_{L}\right) \text { при } r \rightarrow \infty .
$$

Значения $u_{L}$ для фиксированного значения $r_{s}$ являются решениями радиального уравнения Шредингера [14]:

$$
\frac{d^{2} u_{L}}{d r^{2}}+\left[k^{2}-2 V(r)-\frac{L(L+1)}{r^{2}}\right] u_{L}=0 .
$$

Потенциал рассеяния

$$
V(r)=\frac{Z_{i}}{r} \exp \left(-\frac{\alpha}{r}\right)
$$

- экранированный кулоновский потенциал с параметром $\alpha$. Здесь вместо стандартной процедуры вычисления самосогласованного нелинейного потенциала рассеяния методом функционала электронной плотности [12] используется простой потенциал Юкавы. Расчет, однако, усложняется необходимостью оптимизации параметра 


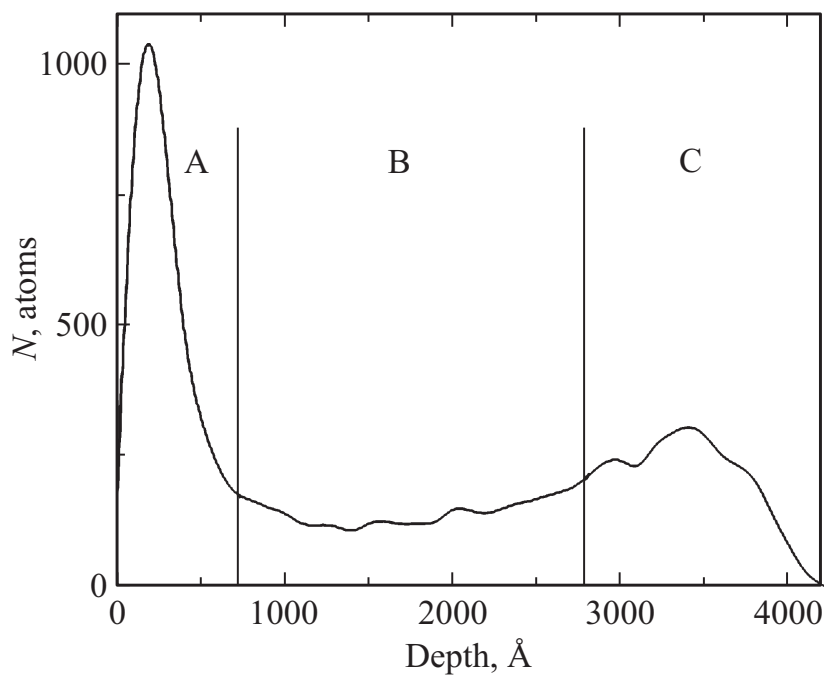

Рис. 2. Профиль распределения ионов $\mathrm{Si}^{+}$по глубине.

экранирования $\alpha$, состоящего в выполнении правила сумм Фриделя для фазовых сдвигов

$$
\frac{2}{\pi} \sum_{L}\left(2_{L}+1\right) \delta_{L}\left(v_{\mathrm{F}}\right)=Z_{i} .
$$

Данные в виде таблиц $S\left(V_{i}, r\right), \mathrm{eV} / \AA$ сохраняются на носителе. Затем, после линейной интерполяции и умножения на длину пробега иона за шаг интегрирования уравнений Ньютона, получаем величину энергии неупругого торможения.

Представленная выше схема неэмпирического расчета торможения медленных ионов является особенно актуальной для полупроводников и диэлектриков. Например, известная нелинейная модель [12] опирается на теорию электронного газа и не подходит для нашего случая. В чистом кремнии имеется небольшая запрещенная зона, и отсутствуют свободные электроны. Следовательно,
Доли ионов (в процентах от общего числа) $\mathrm{Si}^{+}$с $E_{i}=10 \mathrm{keV}$, приходящиеся на области профиля распределения (рис. 2), в зависимости от температуры кристалла $\mathrm{Si}$

\begin{tabular}{c|c|c|c}
\hline$T, \mathrm{~K}$ & А, вне канала & В, выход из канала & С, каналирование \\
\hline 0 & 22 & 18 & 60 \\
300 & 31 & 25 & 44 \\
600 & 34 & 28 & 38 \\
1000 & 38 & 30 & 32 \\
1500 & 42 & 29 & 29
\end{tabular}

неупругие потери иона выступают источником локальных электронных возбуждений, включая образование электрон-дырочных пар.

\section{3. Результаты}

\section{1. Анализ распределения ионов по глубине кристалла}

Профиль распределения ионов по глубине кристалла имеет характерные особенности. На рис. 2 представлен результат расчета для ионов кремния с начальной энергией $E_{i}=10 \mathrm{keV}$ и углом $\alpha=0^{\circ}$ при $T=300 \mathrm{~K}$ (кристалла). Условно можно выделить три области, обозначенные на графике буквами А, В, С.

Область расстояний, обозначенная на рис. 2 буквой А, от 0 до $800 \AA$ соответствует ионам, которым не удалось занять канал. Эти ионы участвовали в жестких столкновениях с атомами решетки и за короткий промежуток времени потеряли энергию. На рис. 3 показано, как изменялась энергия за 1 шаг $(\Delta E)$ со временем $\left(t, \mathrm{fs}=10^{-15} \mathrm{~s}\right)$ для случайно выбранного иона с глубиной пробега $185 \AA$. Последовательность столкновений такова, что в пяти из них ион теряет более $200 \mathrm{eV}$, замедляясь до $900 \mathrm{eV}$ после $125 \mathrm{fs}$ движения.

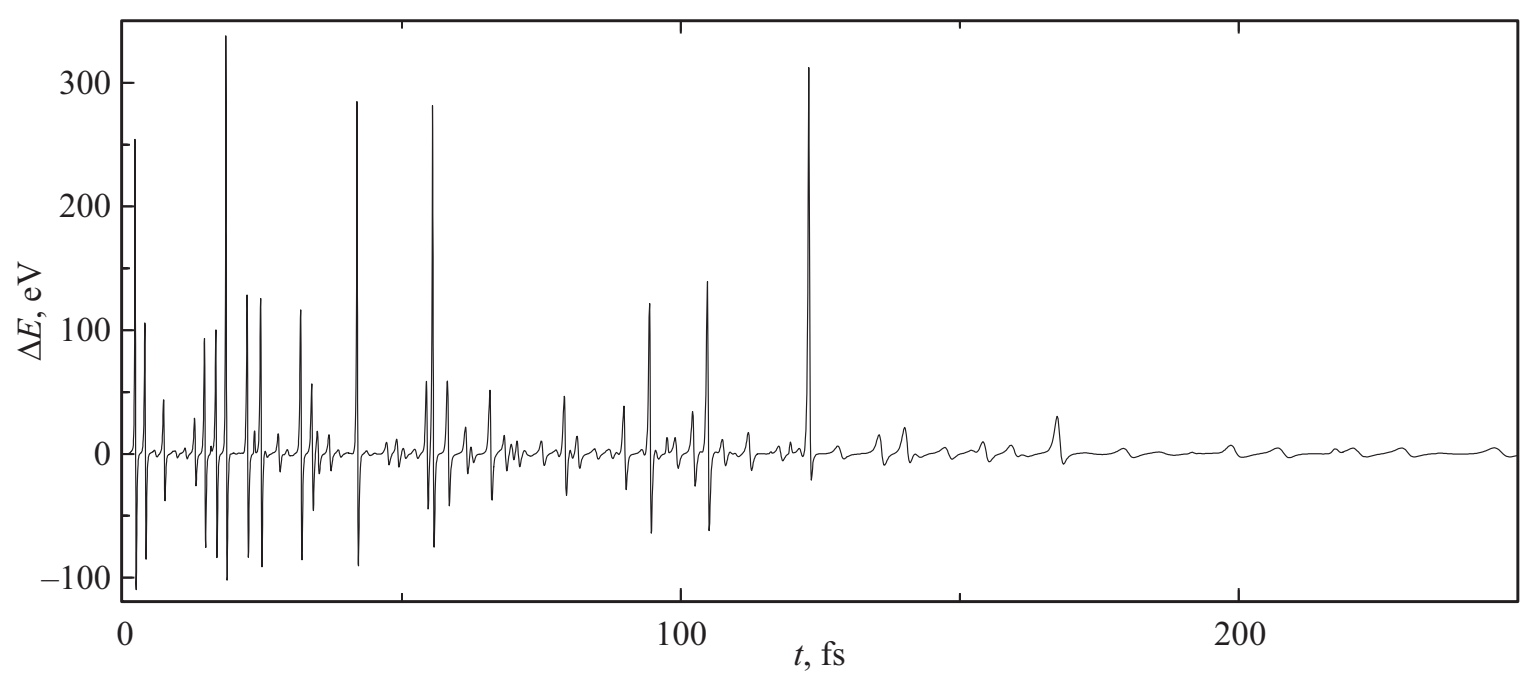

Рис. 3. Потери энергии за шаг ионом $\mathrm{Si}^{+}$, находящимся вне канала. 
Ионы, успешно встроенные в каналы, через определенное время могут испытать сильное столкновение и остановиться. Такие ионы образуют область профиля распределения, обозначенного на рис. 2 буквой $\mathrm{B}, \mathrm{c}$ глубиной проникновения от 800 до $2800 \AA$.

Область С рис. 2, от 2800 до $4000 \AA$, относится к каналированию ионов. Траектория случайно выбранного иона, остановившегося на глубине $3322 \AA$, показана на рис. 4. Зигзагообразная кривая ориентирована вдоль оси $z$ - направления начальной скорости иона.

Экспериментальные данные полностью подтверждают наличие двух максимумов и переходной области В на графиках профиля распределения каналированных ионов. Например, авторы [14] приводят профили, аналогичные рис. 2 , для ионов $\mathrm{P}^{+}$в направлении $\langle 110\rangle \mathrm{Si}$ для $E_{i}$ от 40 до $400 \mathrm{keV}$.

Важно отметить, что приведенные в работе результаты характерны для каналирования так называемых „тяжелых“ ионов $\mathrm{Si}^{+}$. Опыт расчетов легких ионов, например, изотопов водорода, указывает на присутствие лишь одного пика, обозначенного на рис. 2 буквой С. Можно утверждать, что особенности каналирования ионов имеют комплексный характер, который с успехом может быть воспроизведен в рамках моделирования методом молекулярной динамики.

\section{2. Влияние температуры кристалла на профили распределения ионов}

Температура кристалла описывалась с помощью амплитуд колебаний атомов, взаимодействующих между собой посредством многочастичного потенциала $V_{\mathrm{Si}-\mathrm{Si}}$, выражение (2). Начальные отклонения атомов решетки рассчитывались с помощью $T_{\mathrm{D}}$ по формуле (1). Выясним, насколько тепловой шум влияет на каналирование ионов.

В расчетах использовались ионы $\mathrm{Si}^{+}$с $E_{i}=10 \mathrm{keV}$ и углом $\alpha=0^{\circ}$. На рис. 5 приведены профили распределения ионов по глубине для нескольких температур кристалла кремния, $T=0,300,600,1000$ и $1500 \mathrm{~K}$.

Нагрев кристалла препятствует каналированию ионов, переводя все большую часть треков из области С в области А и В, что следует из численных данных в таблице.

Тепловые колебания атомов решетки способствуют увеличению упругого торможения иона, растет вероятность „сильных“ столкновений, выводя ионы из канала. Из смещения пика каналирования влево с ростом температуры, рис. 5, можно сделать вывод об увеличении неупругой компоненты торможения иона. Траектории иона, пересекая области с высокой электронной плотностью, становятся короче.

Из расчетов следует, что скорость деканалирования ионов нелинейно зависит от температуры решетки. Следовательно, условием эффективной реализации каналирования ионов является режим низких температур по сравнению с комнатной.

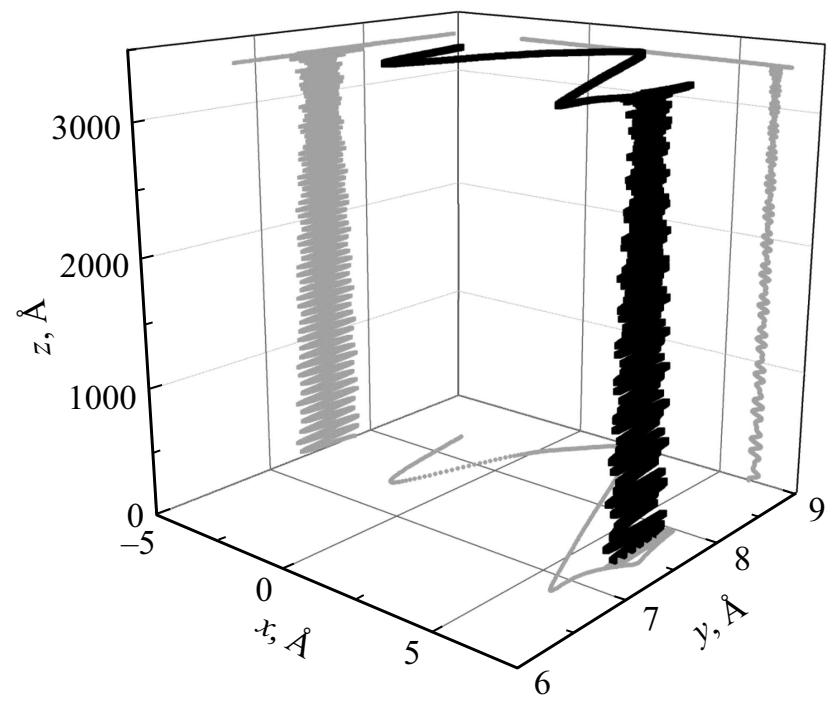

Рис. 4. Траектория иона $\mathrm{Si}^{+}$, двигающегося в канале.

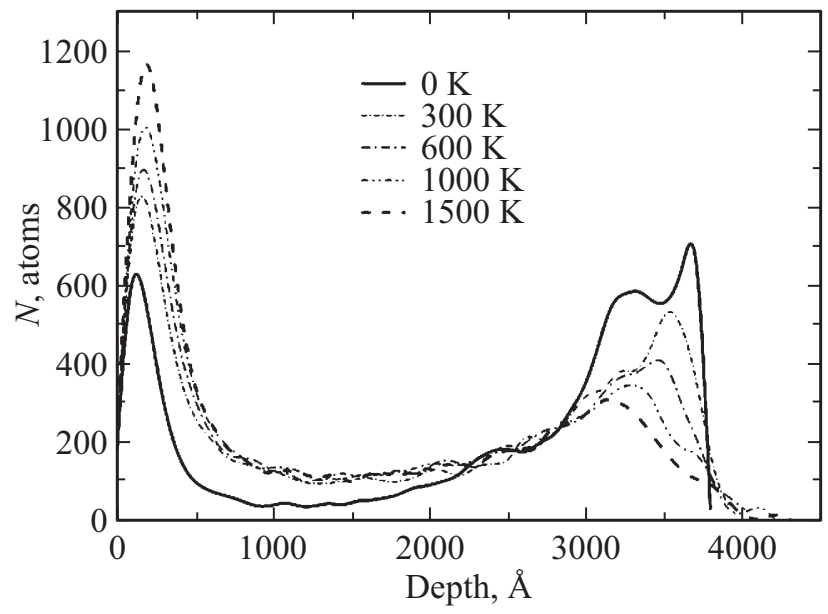

Рис. 5. Температурная зависимость профиля распределения ионов $\mathrm{Si}^{+}$.

\section{3. Влияние угла падения иона на профили распределения}

Совпадение направлений движения иона и симметрии кристалла является основным условием для возникновения осевого каналирования. Малые углы отклонения от оси компенсируются мягким рассеянием на атомах решетки, удерживая ион в канале. Согласно классической статистической теории каналирования [15] существует пороговое значение угла наклона траектории иона к оси, критический угол Линдхарда, при котором ион не может удержаться в канале. Определим с точностью до $1^{\circ}$ значение критического угла с помощью анализа профилей распределения ионов $\mathrm{Si}^{+}$для фиксированной величины начальной энергии $E_{i}$.

Рассмотрим случай, когда ионы кремния с $E_{i}=10 \mathrm{keV}$ проникают в кристалл кремния при $T=0 \mathrm{~K}$ под различными углами к вектору нормали $\langle 100\rangle$ 


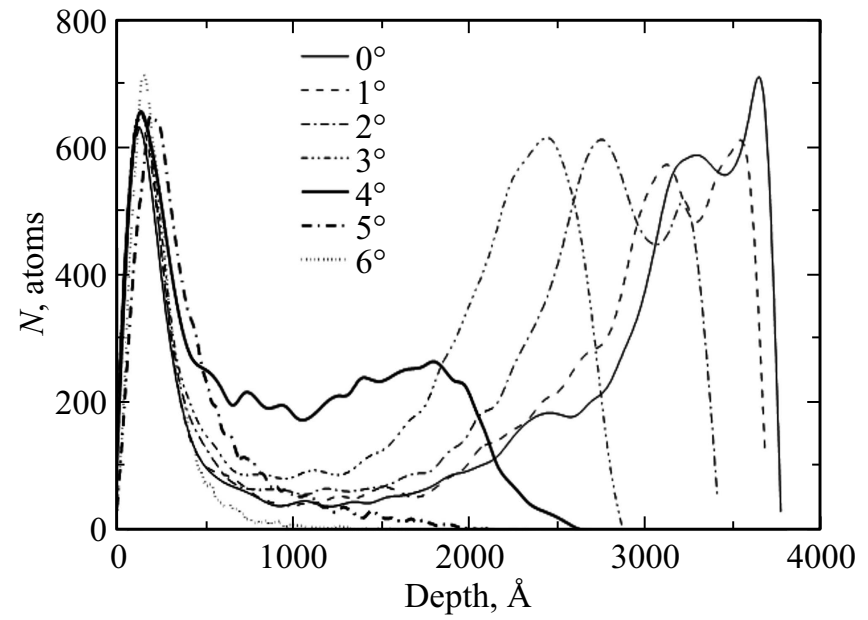

Рис. 6. Определение критического угла Линдхарда по профилям распределения иона $\mathrm{Si}^{+}$с энергией $10 \mathrm{keV}$.

$\alpha=0^{\circ}, 1^{\circ}, \ldots, 6^{\circ}$. Полученные профили показаны на рис. 6.

Пик каналирования на профилях с $\alpha=$ от 0 до $3^{\circ}$ последовательно сдвигается влево, что можно связать с усилением торможения. При достижении угла $\alpha=4^{\circ}$ профиль заметно изменяется. Форма пика каналирования приобретает размытый вид, и высота уменышается приблизительно в 3 раза. Каналирование отсутствует для $\alpha=5$ и $6^{\circ}$, областей В и С на профиле распределения нет. Таким образом, критический угол Линдхарда $\left(\alpha_{c}\right)$ для данных начальных условий равен 4 градусам.

\section{4. Влияние начальной энергии иона на профили распределения ионов}

Из литературы известно [7], что торможение иона нелинейно зависит от энергии. Упругая и неупругая составляющие торможения имеют максимумы приблизительно при энергиях иона 1 и $100 \mathrm{keV} / \mathrm{a} . u$. соответственно. Данное свойство установлено для аморфных структур и кристаллов произвольной ориентации, следовательно, не включает каналирование. Сравним профили распределения медленных ионов и ионов с промежуточной энергией. Для ионов $\mathrm{Si}^{+}$с $E_{i}>400 \mathrm{keV}$ неупругие потери на начальном участке траектории будут рассчитываться по формуле (3), а после замедления до $400 \mathrm{keV}$ по (4). Также выясним зависимость от энергии для профилей ионов с углом падения выше критического. Рассмотрим серию профилей для ионов кремния с начальными энергиями $E_{i}=100 \mathrm{keV}, 500 \mathrm{keV}$ и $1 \mathrm{MeV}$. Температура кристалла $T=300 \mathrm{~K}$, углы $\alpha=0^{\circ}$ и $\alpha=7^{\circ}$.

Для $\alpha=0^{\circ}$ профили распределения отвечают каналированию ионов, рис. 7. Пики распределения имеют крутые склоны, сдвигаясь вглубь кристалла с ростом энергии. Положения пиков распределения имеют близкую к логарифмической зависимость от $E_{i}$, таким обра- зом, согласуются с приведенными выше литературными данными об увеличении неупругого торможения с ростом $E_{i}$ в промежуточном интервале значений.

Иной вид имеют профили распределения для $\alpha=7^{\circ}$, рис. 8. Узкий пик при $E_{i}=100 \mathrm{keV}$ последовательно сдвигается вглубь кристалла и имеет более пологие склоны с ростом энергии. Сравнивая рис. 7 и 8 замечаем, что при высоких энергиях некоторая часть ионов, попадающая в кристалл с $\alpha=7^{\circ}$, участвует в каналировании. Действительно, доля ионов из области пика $46000 \AA$ на профиле $E_{i}=1 \mathrm{MeV}$, рис. 8 , составляет не менее $8 \%$ от общего числа. Таким образом, был сделан вывод о существовании процесса, обратного процессу деканалирования ионов. Для медленных ионов данный процесс не наблюдался.

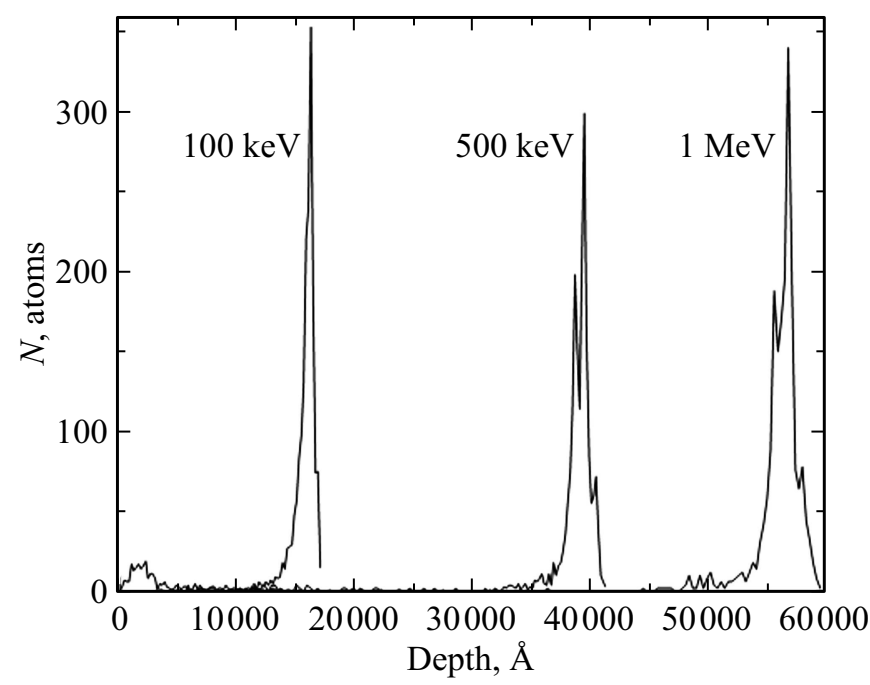

Рис. 7. Профили распределения ионов $\mathrm{Si}^{+}$с высокими $E_{i}$ при $\alpha=0^{\circ}$.

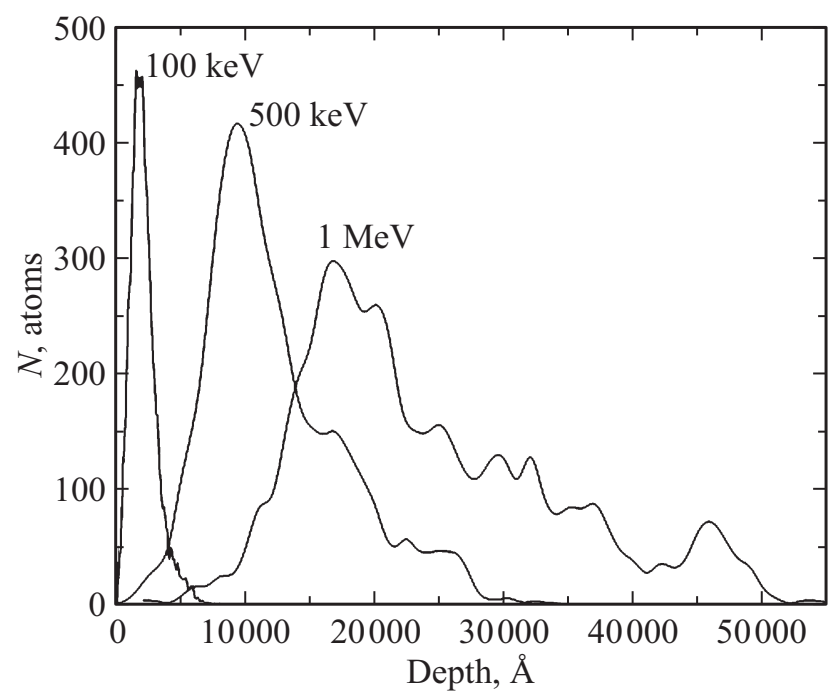

Рис. 8. Профили распределения ионов $\mathrm{Si}^{+}$с высокими $E_{i}$ при $\alpha=7^{\circ}$. 


\section{5. Изотопный эффект}

Роль масс ионов с одинаковым атомным номером (зарядом) в процессах каналирования традиционно не рассматривается. Действительно, статистическая теория [15] не содержит каких-либо зависимостей от массы иона или атома решетки. В работе [16] проводилась проверка положений данной теории путем молекулярнодинамических расчетов, и относительно масс теория была подтверждена. В то же время существуют данные модельных расчетов $[17,18]$, из которых следует „эффект массы“ для критического угла каналирования: $\alpha_{c} \propto \sqrt{M / M_{i}}$, где $M$ - масса атома решетки, $M_{i}-$ масса иона.

Выясним степень влияния масс на каналирование путем сравнения профилей распределения по глубине для ионов кремния ${ }^{28} \mathrm{Si}$ (стабильный) и ${ }^{32} \mathrm{Si}$ (170 лет) с массами 27.977 и 31.974 a.u. соответственно, $E_{i}=10 \mathrm{keV}$ и $\alpha=0^{\circ}$. Пики каналирования ионов расположены близко, и пик ${ }^{32} \mathrm{Si}$ сдвинут вправо на $100 \AA$, рис. 9. Наблюдаемый изотопный эффект связан со свойством инерции ионов. Поскольку при равных начальных $E_{i}$, более тяжелые частицы имеют большие начальные импульсы $\left(p_{i}\right)$, для их изменения требуется приложение большей силы. Изза малой разницы масс рассмотренных изотопов $\mathrm{Si}$ сдвиг пика каналирования невелик. Проведя аналогичные расчеты $\left(E_{i}=10 \mathrm{keV}\right.$ и $\left.\alpha=0^{\circ}\right)$ для ионов изотопов водорода, нами были получены сдвиги пика каналирования: $960 \AA$ для пары ${ }^{1} \mathrm{H}^{+}-{ }^{2} \mathrm{H}^{+}$и $710 \AA$ для пары ${ }^{2} \mathrm{H}^{+}-{ }^{3} \mathrm{H}^{+}$.

В заключении рассмотрим ,эффект массы“ для критического угла каналирования. Как и прежде $E_{i}=10 \mathrm{keV}$, $T=0 \mathrm{~K}$. Критический угол для иона изотопа ${ }^{28} \mathrm{Si}$ был определен $\alpha_{c}=4^{\circ}$ с помощью профилей распределения на рис. 6. Из серии профилей с $\alpha=0^{\circ}, 1^{\circ}, \ldots, 5^{\circ}$, представленной на рис. 10 , находим для ионов ${ }^{32} \mathrm{Si}$ значение $\alpha_{c}=2^{\circ}$. Результат $\alpha_{c}\left({ }^{32} \mathrm{Si}\right)<\alpha_{c}\left({ }^{28} \mathrm{Si}\right)$ служит подтверждением ,эффекта массы“ для критического уг-

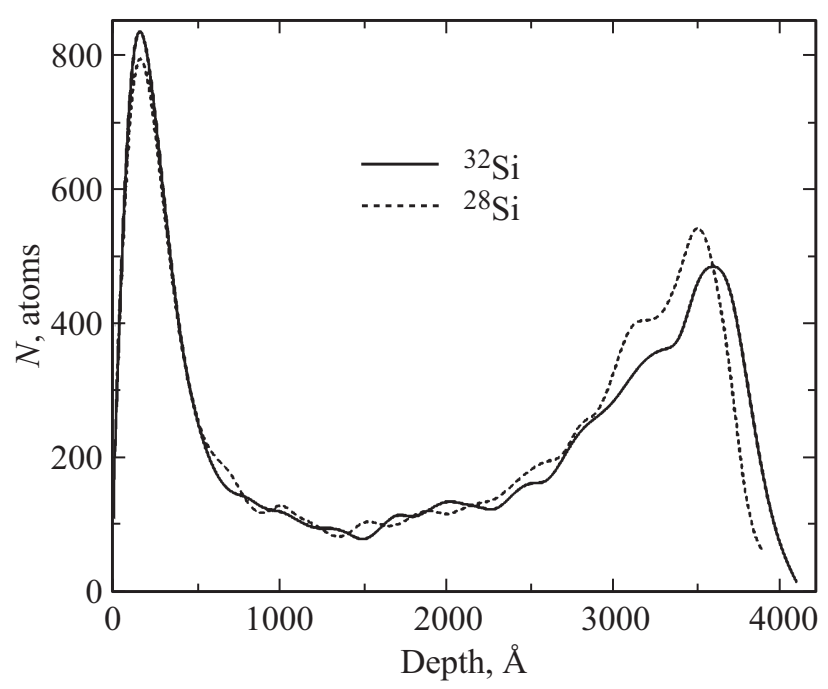

Рис. 9. Профили распределения ионов изотопов $\mathrm{Si}^{+}$при $\alpha=0^{\circ}$.

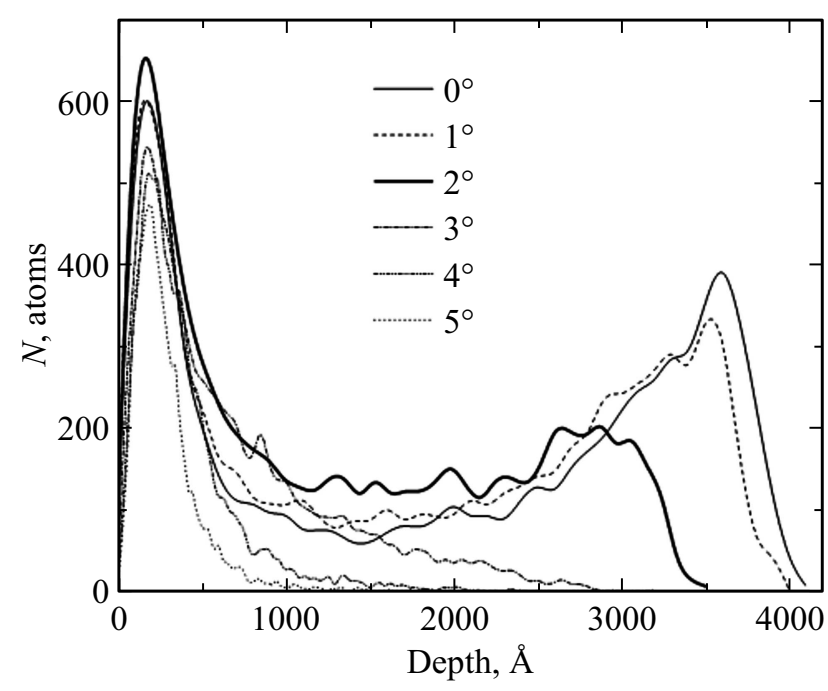

Рис. 10. Определение критического угла по профилям распределения иона ${ }^{32} \mathrm{Si}^{+}$с энергией $10 \mathrm{keV}$.

ла каналирования, и это второе проявление изотопного эффекта.

Демонстрация изотопного эффекта показывает ограниченность статистической теории каналирования, в первую очередь из-за слабого описания динамики процесса. Есть также вопросы к представлению объемной кристаллической структуры в виде цепочек атомов, рассмотрению только одной компоненты энергии иона, перпендикулярной оси канала, и к другим ограничениям.

Вернемся к работе [16] и сделаем необходимые пояснения. Авторы рассчитали каналирование ионов изотопов водорода с $E_{i}=10 \mathrm{keV}$ в направлении $\langle 100\rangle \mathrm{Si}$. Мы провели подобные расчеты и получили хорошее совпадение результатов. Следует подтвердить вывод об отсутствии „эффекта массы“ для ионов изотопов водорода. Но не об отсутствии эффекта как такового. Ионы водорода, благодаря большому отношению $M / M_{i}$ демонстрируют ряд особенностей при каналировании. Анализируя профили распределения ионов водорода видно, что область А (рис. 2) отсутствует, а единственный пик каналирования $\mathrm{C}$ при достижении критического угла скачком перемещается в приповерхностную область. Важно, что „эффект массы“ в $[17,18]$ получен для нескольких сортов ионов, среди которых самый тяжелый имел массу 222 a.u.

\section{4. Заключение}

В работе показано, что процессы каналирования ионов в монокристаллическом кремнии определяются начальными скоростями ионов и температурой решетки. Получено подтверждение изотопной зависимости профиля распределения ионов по глубине и величины критического угла каналирования. Из анализа профилей рас- 
пределения ионов средних энергий с большими углами вхождения следует возможность слабого каналирования.

Каналирование медленных ионов представляет собой сложный физический процесс. Возможно, в будущем будет создана простая и достаточно точная теория каналирования, не требующая серьезных вычислительных затрат. В любом случае необходимо проводить сбор имеющихся экспериментальных и теоретических данных по каналированию ионов, как это уже сделано для аморфных систем авторами [1,7].

Практическое значение медленных ионов, безусловно, будет возрастать. Например, уменьшение размеров элементов микросхем напрямую связано с уменьшением энергии в процессах ионной имплантации. Одновременно необходимо повышать качество профилей распределения примеси по глубине подложки.

За рамками работы остались важные вопросы, связанные с присутствием точечных дефектов кристаллической решетки, тонких эпитаксиальных структур. Не проводился анализ перераспределения поглощенной кристаллом энергии ионов.

\section{Благодарности}

Автор выражает благодарность П.А. Александрову за большую помощь в обсуждении результатов

\section{Финансирование работы}

Работа выполнена при поддержке НИЦ „Курчатовский институт“ (приказ № 1575 от 16.07.2019 г.).

\section{Конфликт интересов}

Авторы заявляют, что у них отсутствует конфликт интересов.

\section{Список литературы}

[1] H. Paul. AIP Conf. Proc. 1525, 309 (2013).

[2] M.T. Robinson, D. K. Holmes, O.S. Oen. In Le Bombardement Ionique (Centre National de la Recherche Scientifique) Paris (1962). 105 p.

[3] G.R. Piercy, F. Brown, J.A. Davies, M. McCargo. Phys. Rev. Lett. 10, 399 (1963).

[4] X. Gonze, F. Jollet, F. Abreu Araujo etc. Comp. Phys. Commun. 205, 106 (2016).

[5] C. Kittel. Introduction to Solid State Physics. Wiley (2004). $704 \mathrm{p}$.

[6] J.F. Justo, M.Z. Bazant, E. Kaxiras, V.V. Bulatov, S. Yip. Phys. Rev. B 58, 2539 (1998).

[7] J.F. Ziegler, J.P. Biersack, U. Littmark. The Stopping and Range of Ions in Matter. SRIM Company (2008). 683 p.

[8] О.Б. Фирсов. ЖЭТФ 36, 1517 (1959).

[9] J. Lindhard, M. Scharff. Phys. Rev. 124, 128 (1961).

[10] P.M. Echenique, R.M. Nieminen, R.H. Ritchie. Solid State Commun. 37, 779 (1981).

[11] J.P. Perdew, K. Burke, M. Ernzerhof. Phys. Rev. Lett. 77, 3865 (1996).
[12] T.L. Ferrell, R.H. Ritchie. Phys. Rev. B 16, 115 (1977).

[13] J. Calera-Rubio, A. Gras-Martí, N.R. Arista. Nucl. Instrum. Meth. Phys. Res. B 93, 137 (1994).

[14] С. Сунакава. Квантовая теория рассеяния. Мир, М. (1979). $272 \mathrm{c}$.

[15] И. Линдхард. УФН 99, 249 (1969).

[16] K. Nordlund, F. Djurabekova, G. Hobler. Phys. Rev. B 94, 214109 (2016)

[17] L.-P. Zheng, Z.-Y. Zhu, Y. Li, D.-Z. Zhu, H.-H. Xia. Nucl. Instrum. Meth. B 266, 849 (2008).

[18] W. Takeuchi. Nucl. Instrum. Meth. B 269, 1355 (2011).

Редактор Ю.Э. Китаев 